\title{
Symbiogenesis and the Early Evolution of Life
}

\author{
Francisco Carrapiço and Telma Rodrigues \\ Universidade de Lisboa, Faculdade de Ciências, Departamento de Biologia Vegetal, Centro de \\ Biologia Ambiental, Bloco C2, Campo Grande, 1749-016 Lisboa, Portugal, e-mail: \\ f.carrapico@fc.ul.pt
}

\begin{abstract}
The concept of symbiogenesis was introduced in 1909 by the Russian biologist Constantin Merezhkowsky as "the origin of organisms by the combination or by the association of two or several beings which enter into symbiosis". In this article we develop this idea, associated to the Freeman Dyson's hypothesis, applied to the early evolutive stages of life, considering that it could be a possible main rule in the appearance and development of life conditions on Earth and elsewhere. A cooperative, synergistic strategy should be considered as having been the determinant in the development of the survival of the fittest, especially under extremely adverse environmental conditions. This concept must be also applied to the first communities of cells as the base supporting evolution of the early "tree of life". Cells, like we have previously described, can be included in a new cellular concept entitled, "symbiocell", since survival of the community under such adverse conditions required a cooperative, synergistic strategy. Similar principles could also be used to understand chemical pre-biotic evolution. We believe that astrobiologists should consider it as a new approach to understand organic and biological evolution.
\end{abstract}

Keywords: origins of life, symbiogenesis, evolution

\section{INTRODUCTION}

In his classic 1941 paper "Astrobiology", Laurence J. Lafleur discusses the conditions of the origin of life in the universe, pointing out that "the process of evolution depends upon de gradual accretion of relative minute variations or mutations, and the occurance of these evolutionary elements is recognized to be a matter of chance". The author also discusses the problem in a broader multitude approach than the quoted phrase, concluding "that life in the universe is not confined to our planet". Even if it was written in the 40's, this perspective, related to the problem of origin of life in the universe, represents an exception to the major scientific thought of that time and the rest of the century. It also includes, however, some limitations to the subject, not only in the scientific and technological knowledge, as expected, but also in the epistemological way we must understand the evolution of life. Based on the neo-Darwinian theory that is the main approach we can offer when we look at the origin of life. Still, we believe that other approaches could be implemented, namely using symbiogenic principles. It is on this new vision that this paper will focus.

\section{THE SYMBIOGENIC APPROACH}

The origin or origins of life is still an open problem. Usually we consider Aleksandr Oparin and John Haldane's ideas, published in 1924 and 1929, respectively, as the main sources for the development of the modern thinking on the origins of life, but it was Constantin Merezhkowsky, a Russian biologist, who in 1909 in the work "The Theory of two Plasms as Foundation of Symbiogenesis, New Doctrine on the Origin of Organisms" pointed out the importance of extremophiles and extreme environments in the early stages of life on Earth ${ }^{2}$. In that paper Merezhkowsky introduces, for the first time, symbiogenic principles to understand the evolution of life under extremelly adverse environmental conditions. This scenario, reconsidered in recent years, is the base of a new paradigm to understand organic and biological evolution on Earth and eventually elsewhere. It was also in that article that Merezhkowsky introduced the symbiogenesis concept, defined as "the origin of organisms through the combination or association of two or more

Astrobiology and Planetary Missions, edited by Richard B. Hoover, Gilbert V. Levin, Alexei Y. Rozanov,

G. Randall Gladstone, Proc. of SPIE Vol. 5906, 59060R, (2005) $\cdot 0277-786$ X/05/\$15 · doi: 10.1117/12.617564 
beings that enter in symbiosis". According to this concept, symbiogenesis should be understood as an evolutive mechanism and symbiosis as the vehicle, through which that mechanism unfolds. This fact represents a point of view which is opposed to that of the neo-Darwinism or Modern Synthesis Theory. Thus, according to today's dominant theory, evolution is a gradual process essentially consisting of a natural selection conducted on minimal phenotypical variations, which are a product of genetic and chromosome exchange. To put it in a nut shell, is the development of life a saltational symbiotic process or a gradual process, oriented by natural selection and leading organisms to adaptation? Especially since 1859, the year of the publication of Charles Darwin's book "On the Origin of Species", evolution has been considered as the fundamental concept and organizing factor of modern Biology, as well as its structural pillar. Without denying many of the Darwinist principles, the worse thing we could do within the study of the process of evolution would be to mix up or limit evolution to the Darwinist or neo-Darwinist perspective. These points of view are mainly used to explain the biological evolution, contributing to the generalized belief that evolution could be explained by these two scientific theories. This led to the erroneous idea that Darwinism, or neo-Darwinism, are synonyms of biological evolution. Other evolutionist approaches exist and it is necessary for them to be deepened and discussed within biologic sciences. In this sense, we would like to bring to your attention a set of principles and data that could be integrated in a Symbiogenic Theory of Evolution. This theory must include Darwinist principles, but does not limit itself to the latter in its attempt to promote and explain the development, organization and evolution of the biological world in a symbiogenic sense. However, this approach does not present the cooperative perspective as the only leitmotif in its explanation of the biologic phenomena. Consequently, the concept of symbiosis, which we consider as the most valid to understand the biologic and evolutionary phenomena, is the one that was developed and presented by Anton de Bary in 1878 and defined as "the common life of different organisms"3. The latter does not imply a strict compartimentation of interspecific relationships, thus it should be regarded as a continuous and dynamic process of different relations, such as mutualism, parasitism and commensalisme. In this process, the acquisition of new genes through lateral transfer plays an important role. The same applies to the development of new metabolic capacities acquired by an organism from other organisms associated to it. The existence of mutual benefit should, however, not be considered, as the plus or common denominator of the symbiotic process. As it was adequately referred by Dubos and Kessler, in 1963, during the $1^{\text {st }}$ International Conference on Symbiosis, in London, "the nutritional effects of symbiosis are not its most interesting manifestation. More remarkable is the fact that many symbiotic systems produce substances and structures that neither one of the two components produces when growing alone"4.

In what way could this approach help us to better understand the origin of life on Earth or elsewhere? The OparinHaldane ideas constitute the main principles of the heterotrophic hypothesis of the origin of life and were reinforced by the successful results of the 1953 Miller-Haldane experiment. The Oparin-Haldane hypothesis rest on the assumption that the mixture of gases in Earth's early atmosphere was much different from today. Large amounts of these gases would have dissolved in the primitive oceans and would have received energy from sunlight, lightning, and the Earth's internal heat. Both authors suggested how this energy could have caused the simple molecules to combine into more complex molecules and how the latter could have formed larger and larger combinations. After several hundred million years, such combinations could have formed the first living cells, following a Darwinian padron of evolution in which the survival of the fittest and natural selection were the main driving rules for the appearance of life on Earth. Even with some modification, these approaches are the principal theoretical tools that present scientists can use to probe the formation of life in the astrobiological domain. However, there is a great difference between the Oparin's and Haldane's approaches. It is related to the two fundamental functions of life - metabolism and replication - . While Oparin gave an evolutionary priority to metabolism, Haldane gave it to replication, and the choice between these two alternatives still divides, nowadays, the fields of the theories of origin of life ${ }^{5}$.

Both these two theories are built on the belief that evolution was a Darwinian strict process without any cooperative or synergistic approach. However, we believe that this approach must evolve in a more symbiogenic way, introducing new concepts that enable us to understand the natural world in a more cooperative sense and eventually closer to reality. In this sense, the conventional "survival of the fittest" cannot be applied in that traditional way. A cooperative, synergistic strategy should be considered as having been the determinant in the development of the survival of the fittest, especially under extremely adverse environmental conditions. One good example is the stromatolites, considered as the first ecological strategy evolved for survival on primitive Earth, not for one organism but for a community of organisms. This view can also be applied to the pre-biotic evolution. In 1983, Freeman Dyson presented a hypothesis for the origin of life using symbiogenic concepts, which is further developed in his book "Origins of Life" published in $1985^{6}$. He invoked Lynn Margulis' ideas on the origin of eukaryotic cells, proposing that the pre-biotic evolution would have been 
accomplished by the independent formation, on one hand, of metabolic systems, and on the other hand, of autoreplicative molecules. At a specific moment, some of these molecules would have been synthesized within some of those systems, first as parasites and then as symbionts, which evolved together. Under this concept, the primitive nucleic acids, namely RNAs, invaded their metabolic hosts and used them for their own replication, in a schema summarized "metabolism first, replication second". Although, in these circumstances, the replication of nucleic acids could be unlikely, it gives an interesting solution to the main dilemma, how primitive systems could be able to storage information and to perpetuate themselves, using nucleic acids. However, in terms of biochemical principles, the replication process seems to be simpler than the metabolism one, which leads us to the belief that this process could have had the leading role in this evolutive scenario. But as Marcello Barbieri points out, "it remains to be seen, however, if the replication paradigm can really account for the processes that led to the origin of the first cells."

Following the replication paradigm, the formulation of the so called "RNA world" by Walter Gilbert in 1986 to designate a hypothetical stage in the development of life in which RNA was the primary information and catalytic molecule, combining the properties of RNA and proteins, was a natural theoretical step after the discovery of ribozymes by Thomas Cech and Sidney Altman. The fact that ribozymes came before protein enzymes does not mean that replication came before metabolism, although it was generally interpreted that if RNAs could behave as genes and enzymes, they became the first replicators in the history of life ${ }^{5}$. According to this scenario the RNA world should be a world of replicators, implying that primitive Earth was populated by RNAs showing the capacity to replicate themselves or to catalyse the replication of other RNAs". This has not been proven yet. Furthermore, RNAs are "sophisticated, evolutionarily advanced molecules", ${ }^{\text {", }}$ which could imply that the latter were not the main leading molecules responsible for the chemical evolution on primitive Earth. In this sense, we would like to think that a symbiogenic scenario was responsible for the development of pre-biotic processes that led to the origin of primitive cells.

\section{CHEMICAL BLOCKS OF LIFE AND PRIMORDIAL CELLS}

The establishment and development of cellular life needs the presence of the adequate molecules for the chemical and organic evolution. The nature and origin of these chemical blocks of life are essential to understand how life had been formed and how it has evolved. Two main scenarios exist to explain its origins: the endogenous and exogenous models. The first one includes the theoretical Oparin-Haldane hypothesis, including the Miller-Urey experiment and the hydrothermal-systems hypothesis. The second one is associated to extraterrestrial synthesis and its delivery to Earth by cosmic bodies, such as comets and meteors. The first protocells were membrane-bounded systems of catalytic and replicating macromolecules ${ }^{8}$, similar to the models presented by Oparin and Sidney Fox (coacervates and microspheres). The formation of these protocellular systems begins, in our perspective, with the self-assembly of primitive membranes in aqueous environments creating a barrier and defining the separation between the outside and inside medium. These membranes could be formed spontaneously in the prebiotic milieu or even in some cosmic bodies. The presence of amphiphilic compounds isolated from the Murchison meteorite, having the ability to self-assembly into membrane vesicules $^{8}$, could indicate a possible path on how these primitive systems could be formed and evolve. In these cases, life in prebiotic environmental conditions will involve the development of cellular systems of encapsulated replicating/catalytic macromolecules ${ }^{8}$.

In his article "The Universal Ancestor", Carl Woese presents a genetic model for the universal ancestor of extant life, pointing out that "the universal ancestor is not a discrete entity. It is, rather, a diverse community of cells that survives and evolves as a biological unit". He named this universal ancestor progenote. We believe that this theoretical scenario fits correctly into the idea of a cell community that existed and evolved in the early life stages on Earth. On this sense, the traditional concept of "primordial cell" is a biological chimera in our point of view. We suggest there were probably open communities of protocells, changing and incorporating information among them, as the base supporting evolution of the early "tree" of life. Ultimately, we consider that primitive reality to a huge "prebiotic web library". From this point of view, one important question must be formulated. Did those protocells evolve from elements present only on Earth, or did they incorporate alien materials? Even if we still do not have a final answer, both scenarios should always be presented in any symbiogenic strategy. Cells, like we have previously described, can be included in a new cellular concept entitled, "symbiocell", since survival of the community under such adverse conditions required a cooperative, synergistic strategy. A "symbiocell" is a concept based on the principles that the cell or the protocell is organized not only as a separate biological unit, but as a community of functions acquired by symbiogenic means and evolved using

Proc. of SPIE Vol. 5906 59060R-3 
symbiotic rules. In a sense, evolving and behavioring like an ecological community or a natural microcosm. The "symbiocell" will be the material support for the existence and development of the adequate information flow, energy and metabolites that constitute the web base of the autonomous and further communal life evolution. Later, these cells continue to evolve in a symbiogenic way, involving and acquiring specialized microbial communities which lead to the appearance of the eukaryotic cells, according to the serial endosymbiotic theory ${ }^{10}$.

\section{FINAL REMARKS}

In this paper we have used a new approach to understand the problem of the origin of life on Earth. According to this point of view, we believe that the metabolism/replication dilemma is not the main issue to be considered. The main point will be how evolution took place and worked in such early adverse environment. To obtain a good answer to this problem, we must consider different evolutive approaches, other than the traditional Darwinian ones, introducing new tools to build a symbiogenic scenario of evolution. A cooperative and a communal synergistic strategy should be considered as having been the determinant in the development of the "survival of the fittest", especially under such extremely adverse environmental conditions. This approach could also be used to understand the life formation in other locations beyond our planet.

\section{ACKNOWLEDGEMENTS}

The authors are grateful for the technical assistance of Maria Helena Costa and Helena Carrapiço.

\section{REFERENCES}

1. L.J. Lafleur, “Astrobiology”, Astronomical Society of the Pacific. Leaflet №. 143, 333-340, 1941.

2. J.Sapp, F. Carrapiço and M. Zolotonosov, "Symbiogenesis:The Hidden Face of Constantin Merezhkowsky", Hist. Phil. Life Sci., 24, 413-440, 2002.

3. A. de Bary, "Ueber Symbiose", Tageblatt 51. Versamml. Deutscher Naturforscher u. Aerzte, Cassel, 121-126, 1878.

4. R. Dubos and A. Kessler, "Integrative and Disintegrative Factors in Symbiotic Associations", Proceedings of the Thirteenth Symposium of the Society for General Microbiology, Nutman, P.S. \& Mosse, B. (eds.), p. 1-11, London, 1963. 5. M. Barbieri, "The Organic Codes". An Introduction to Semantic Biology", Cambridge University Press, Cambridge, 2003.

6. F. Dyson, "Origins of Life", Cambridge University Press, Cambridge, 1985.

7. S.L. Miller, "Which Organic Compounds Could Have Occurred on the Prebiotic Earth?", Cold Spring Harbor Symposia on Quantitative Biology, 52, 17-27, 1987.

8. D.W. Deamer, E.H. Mahon and G. Bosco, "Self-assembly and Function of Primitive Membrane Structures", in E. Bengtson (ed.) Early Life on Earth, Nobel Symposium No. 84, Columbia U.P., New York, pp. 107-123, 1994.

9. C. Woese, "The Universal Ancestor", Proc. Natl. Acad. Sci. USA, 95, 6854-6859, June 1998.

10. L. Margulis, Origin of Eukaryotic Cells: Evidence and Research Implications for a Theory of the Origin and Evolution of Microbial, Plant and Animal Cells on the Precambrian Earth. Yale University Press, London, 1970. 